Med Klin Intensivmed Notfmed 2014 .

109:34-40

DOI 10.1007/s00063-013-0304-5

Eingegangen: 17. September 2013

Angenommen: 12. November 2013

Online publiziert: 4. Januar 2014

๑) Springer-Verlag Berlin Heidelberg 2014

Redaktion

M. Buerke, Siegen
H. Burchardi

Bovenden

\section{Intensiv- und Palliativmedizin}

\section{Von der akademischen Distanz zur fürsorglichen Zuwendung}

\begin{abstract}
Der Intensivmediziner ist während seines aktiven Berufslebens meist im turbulenten Tagesablauf gefangen. Er bemüht sich, den immer schwierigeren Anforderungen gerecht zu werden. Sein Blickwinkel wird dabei vom eigenen Handlungsbereich bestimmt. Nach Ende einer langen Berufslaufbahn als aktiver Intensivmediziner bietet sich mir nun eine neue Wahrnehmung von außen. Das gibt Gelegenheit, den Blickwinkel des Patienten oder eher des Angehörigen einzunehmen. Aus diesem neuen Blickwinkel stellt sich vieles anders dar und die Gewichte verschieben sich. Die folgenden Überlegungen sind keine kritische Analyse, sondern eher ein gefühltes Unbehagen aus täglichem Erleben. Sie sollen uns Intensivmediziner anregen, zu einer menschlicheren Intensivmedizin zu kommen.
\end{abstract}

\section{Situation}

Jährlich versterben in Deutschland etwa 45.000 Menschen auf Intensivstationen. Wir sind uns sicher alle darüber einig, dass die meisten lieber zu Hause sterben würden. Aber das ist für viele nicht zu erreichen. Das Problem ist also nicht selten; es ist auch unser Problem und wir müssen uns damit beschäftigen.

Um das komplexe Zusammenspiel $\mathrm{zu}$ verstehen, müssen wir die unterschiedlichen Situationen der Betroffenen beleuchten:
Da ist zunächst die Situation des Intensivmediziners: Intensivmedizin ist stets durch die hohe Dringlichkeit, die unklare Ausgangslage, den kontinuierlichen Wechsel der Situationen und die immerwährende Ungewissheit des Erfolges gekennzeichnet. Andererseits verfügen wir heute über immense technische Möglichkeiten, die wir zur Diagnostik und Therapie einsetzen können. Überspitzt ausgedrückt: Der Intensivmediziner kann alles und muss alles. Und wenn er es nicht schafft, dann hat er das Gefühl, versagt zu haben.

Gleichzeitig steht der Intensivmediziner unter typischen beruflichen Zwängen: Da ist der sog. technologische Imperativ, der uns veranlasst, alles denkbar Mögliche zu tun, ohne dass wir das Endergebnis, eventuelle Folgeschäden oder gar Kosten bedenken. Das ist für uns insbesondere dann naheliegend, wenn der $\mathrm{Pa}-$ tient ohne Intensivtherapie wahrscheinlich sterben würde, oder wenn er selber keine eigene Entscheidung fällen kann, und wir diese für ihn übernehmen müssen. Auch fürchten wir uns vor den strikten Rechtsvorschriften zu Kunstfehlern und den Haftungsverpflichtungen für Folgeschäden und werden $\mathrm{zu}$ einer Defensivmedizin provoziert [15], die hohe Anforderungen an Diagnostik und Therapie stellt und (u. U. unnötige) hohe Kosten verursacht. Zudem sind wir Intensivmediziner prozessorientiert, d. h. wir orientieren uns nach dem Heilerfolg und denken weniger an die spätere Lebensqualität des Patienten.
Im Umgang mit unseren Patienten und deren Angehörige kommt noch etwas Weiteres hinzu: Technische Befunde haben heute (besonders in der Intensivmedizin) hohe Bedeutung. Das führt dazu, dass wir Ärzte sie auch besonders wichtig nehmen. Dadurch verstärken wir den Eindruck der sog. Apparatemedizin. Ein Ausdruck, den ich für unglücklich halte und der auch falsch ist, da nirgendwo in der Klinik sich so viele Menschen um die Kranken kümmern wie auf der Intensivstation. Doch wir können diese Fürsorge offenbar nicht überzeugend vermitteln.

Die Angehörigen schließlich haben ihre eigenen Sorgen: Die Sorge über die schwere Krankheit ihres lieben Menschen. Und in lebensbedrohlicher Situation haben sie Sorge, dass nicht alles getan wird, was möglich wäre (womöglich aus Gründen des Kostendrucks). Noch mehr aber fürchten sie sich oft vor unserer sog. Übertherapie, die keine Grenzen findet, die nicht aufhören kann.

Dies alles müssen wir ernst nehmen und mir scheint, dass wir dieses erst lernen müssen. 


\section{Wandel der Zeiten}

Wie ist das alles entstanden? Wo kommen wir her?

Lassen Sie uns ein wenig in Zeiten zurückblicken, in denen die Medizin noch fundamental anders war: Früher, in den vergangenen Jahrhunderten, war die Medizin karitativ. Sie konnte nicht viel heilen. Aber sie kümmerte sich um das Leiden der Menschen, so gut sie eben konnte, - auch wenn der Arzt oder die Schwester nur die Hand des Kranken hielt oder ihm einen Trank reichte. Damals bestand die ärztliche Kunst im Zuhören und im Mitfühlen. Sind wir nicht seinerzeit vielleicht gerade deswegen Ärzte geworden?

Erst in dem vergangenen Jahrhundert kam es dann zu den immensen Fortschritten der kurativen Medizin: Mit der Entwicklung der Hygiene und der Antibiotika konnten die Seuchen und Infektionen erfolgreich behandelt werden. Die insuffizienten Organfunktionen wurden durch Beatmung, Nierenersatzverfahren und vieles Andere mehr vorübergehend überbrückt oder die defekten Organe sogar durch Transplantationen ersetzt. Die ehemals hohe Säuglingssterblichkeit wurde durch intensive Therapiekonzepte deutlich reduziert, ... um nur Einiges zu nennen. All das hat dazu beigetragen, dass sich die Lebenserwartung von 1875-2005 (also in nur 130 Jahren) immerhin nahezu verdoppelt hat.

Die Medizin hat sich also fundamental gewandelt: Es ist der Wandel von der karitativen Fürsorge zur kurativen Therapie. Und hier hat insbesondere auch die Intensivmedizin ihre hervorragende Bedeutung bewiesen. Viele Erfolge wären gar nicht möglich ohne die Intensivmedizin.

\section{Ein Wandel unseres Selbstverständnisses?}

Kann es nicht sein, dass dieser Erfolg auch uns verändert hat? Vielleicht hat sich damit das Selbstverständnis der Ärzte gewandelt: Mit der Begeisterung über unsere Erfolge haben wir möglicherweise unsere Empathie mit dem leidenden Menschen aus dem Auge verloren.
> Wir sind heute die Sachwalter der Krankheit, nicht des kranken Menschen.

Der Sachlichkeit gilt unser Hauptaugenmerk. Das wird natürlich in der Intensivmedizin besonders deutlich, wo wir mit dem sedierten Patienten meist ohnehin nicht sprechen können. Wo wir uns um die komplexe Pathophysiologie des Multiorganversagens, die unübersichtlichen Zusammenwirkungen der unterschiedlichen Krankheitszustände, die zahlreichen medikamentösen Interaktionen usw. sachlich kümmern müssen. Der Patient selber kann dazu gar nichts beitragen, er ist Objekt, nicht Subjekt.

\section{\ Intensivmedizinische Behandlung ist durch konzentrierte technische Sachlichkeit bei ärztlicher Sprach- losigkeit gekennzeichnet.}

Gefühle lassen wir gar nicht erst aufkommen; sie stören nur die sachlich Aufgabenerfüllung. Vielleicht haben wir es sogar erst mühsam lernen müssen, möglichst gefühlsneutral zu handeln. Das könnte schon eine „déformation professionelle" geworden sein. Überspitzt gesagt sehen wir unsere Aufgabe darin, einem Dienstleistungskunden einen reibungslosen Service zu bieten.

Hier also liegen in der Medizin Segen und Fluch eng beieinander: Unsere Prioritäten haben sich offenbar gewandelt: Heilen steht an oberster Stelle, - doch was kommt danach?

\section{Erfolg und Misserfolg}

Die Erfolge der Medizin waren in den vergangenen 100 Jahren enorm, und wir dürfen mit Recht darauf stolz sein. Doch eines dürfen wir nicht vergessen: In gleichem Maße sind wir auch für unsere Misserfolge verantwortlich, für die Nebenwirkungen der Intensivmedizin.

Und als Misserfolge werte ich hier die zu lange Behandlung ohne Aussicht auf Erfolg, das „Nicht-Aufhören-Können“. Durch diese Nebenwirkungen der Intensivmedizin wird für viele unserer Patienten das entsetzliche Leiden verlängern.

\section{D) Wir sind in der \\ Intensivmedizin auch für unser "Nicht-Aufhören- \\ Können" verantwortlich}

Wir kennen doch alle die Situation, dass wir mit unserer Behandlung nicht aufhören können, obwohl wir eigentlich innerlich schon das ungute Gefühl haben, dass es ohne Erfolg sein wird. Wir sind auch dafür verantwortlich, dass wir kein Ende finden können. Das steht in unserer Negativbilanz. Es reicht nicht, dass wir uns resignierend sagen, dass der erwartete Erfolg leider nicht eingetreten ist. Vielleicht sind wir auf dem Weg unseres Erfolgs ja ein wenig überheblich geworden. Viele von uns empfinden den Tod eines Patienten als eine persönliche Niederlage: Schließlich haben wir in unserem gesamten Berufsleben gegen den Tod in seiner ständigen Präsenz gekämpft. Er ist unser Feind schlechthin. Und nun haben wir doch verloren. Es ist schwer, sich diese Erkenntnis einzugestehen.

Aber gerade hierin liegt unsere Beschränktheit. Wir müssen bescheidener werden, müssen wieder demütig werden. Wir müssen auch als Ärzte lernen loszulassen. Wir wissen nur zu gut, wie schwer das ist.

\section{Rückbesinnung}

So wäre jetzt also eine Rückbesinnung nötig. Wir müssen endlich erkennen und akzeptieren, dass uns Grenzen gesetzt sind, dass durch unser Tun der Tod natürlich nur verzögert, nicht besiegt werden kann. Dabei haben wir doch heute für unsere therapeutische Ausweglosigkeit einen überzeugenden neuen Weg mit der Palliativmedizin.

\section{Palliativmedizin}

Wenn wir den Patienten mit unserer Intensivmedizin nicht mehr heilen können, dann bedeutet das heute eben nicht das Ende unserer Hilfe. Es greift die Palliativmedizin, wenn wir mit unserer Intensivmedizin keinen Heilerfolg mehr sehen.

Wir wechseln nur das Therapieziel von der kurativen Heilmedizin zur kari- 
tativen Fürsorgemedizin [13]. Der Auftrag ist jetzt: Schmerzbehandlung, Stillen von Hunger- und Durstgefühlen, Körperpflege, Sensibilisierung von Bedürfnissen u. v. a. m. Dazu kommt menschenwürdige Unterbringung, Angehörigenbetreuung, psychosoziale Hilfe: also intensive Zuwendung.

Das bedeutet aber für die moderne Medizin ein wirklicher Paradigmenwechsel. Es ist als fände die Medizin wieder zurück zu ihrer ursprünglichen karitativen Aufgabe. Unbedingt muss der Eindruck vermieden werden, die Ärzte hätten den Patienten „aufgegeben“. Wie fatal ist die Bemerkung: „Ich kann nichts mehr für ihn tun“.

Ich denke, dass Intensivmedizin und Palliativmedizin zusammen gehören; sie sind einfach 2 Seiten einer Medaille [11]. Wenn diese Partnerschaft zustande käme und gelebt würde, dann hätte die Intensivmedizin ihr menschliches Antlitz gefunden, - hätte das Verdikt der Apparatemedizin überwunden. Palliativmedizin ist in Deutschland auf dem Vormarsch. Eine internationale Charta nimmt uns alle in die Pflicht [12]: in gutes Zeichen.

\section{Therapiezieländerung}

Im Jahr 2004 hat die Bundesärztekammer in der ersten Fassung der „Grundsätze zur ärztlichen Sterbebegleitung “ den Begriff der Therapiezieländerung, i. e. die Änderung der Behandlung von der kurativen Zielsetzung der Heilung hin zu dem karitativen Ziel der Leidensverminderung, eingeführt [4].

Ich glaube, das war ein ganz wichtiger Schritt: Durch den Begriff wurde deutlich gemacht, dass der ärztliche Auftrag auch dann nicht beendet ist, wenn keine Heilung mehr zu erwarten ist. Es ändert sich also nur das Ziel: Die ärztliche Fürsorge ist weiterhin geboten.

\section{》) Trotz Therapiezieländerung \\ ist die ärztliche Fürsorge weiterhin geboten}

Diese wichtigen Grundsätze wurden dann im Jahr 2011 nochmals weiter ergänzt und bieten jetzt eine gute Basis für das Handeln der Ärzte [5]. Das Selbstbestim-

Med Klin Intensivmed Notfmed 2014 • 109:34-40 DOI 10.1007/s00063-013-0304-5

(c) Springer-Verlag Berlin Heidelberg 2014

\section{H. Burchardi \\ Intensiv- und Palliativmedizin. Von der akademischen Distanz zur fürsorglichen Zuwendung}

\section{Zusammenfassung}

An den großen Erfolgen der kurativen modernen Medizin hat die Intensivmedizin erheblichen Anteil. Dabei scheint allerdings die empathische Zuwendung zum Patienten und zum Angehörigen zu kurz zu kommen. Unter dem Zwang zur Sachlichkeit und Effizienz sowie dem ökonomischem Druck ist der Intensivmediziner zum Sachwalter der Krankheit, nicht aber des kranken Menschen geworden. Er kämpft gegen die Krankheit und empfindet den Tod seines Patienten als persönliche Niederlage. Dabei muss auch der Intensivmediziner in therapeutischer Aussichtslosigkeit das "Loslassen“ lernen: Er ist genauso für eine unsinnige Übertherapie verantwortlich wie für die Erfolge seiner Behandlung.

Heute gibt es im Fall einer Änderung des Therapieziels bei aussichtsloser Intensivbe- handlung die Möglichkeiten der karitativen Fürsorge durch die Palliativmedizin. Diese ist dann die konsequente Weiterführung der Intensivbehandlung. Die letzte Lebensphase auf der Intensivstation erfordert wiederholte Gespräche mit den Angehörigen und intensive Zuwendung, die in einer zunehmend ichbezogenen Gesellschaft immer seltener wird. Die Kultur der Zuwendung sollte jedoch auch im Behandlungsteam der Intensivstation gepflegt werden. So kann die Intensivstation ein Ort der Menschlichkeit werden und bleiben.

\section{Schlüsselwörter}

Medizinische Sinnlosigkeit .

Entscheidungsfindung · Empathie .

Beratung $\cdot$ Behandlungsteam

\section{Intensive and palliative care medicine. From academic distance to caring affection}

\section{Abstract}

Intensive care medicine has made great contributions to the immense success of modern curative medicine. However, emotional care and empathy for the patient and his family seem to be sparse. There is an assumed constraint to objectivity and efficiency, as well as a massive economic pressure which transfers the physician into an agent of the disease instead of a trustee of the ill human being. The physician struggles against the disease and feels the death of his patient as his personal defeat. However, in futile situations the intensivist must learn to let go. He is responsible for futile overtreatment as well as for successful treatment.

Today, in futile situations in the intensive care unit (ICU), it is possible to change the goal from curative treatment to palliative care. This is a consequent further development from critical care medicine. In end-oflife situations in the intensive care unit, emotional care and empathy are mandatory using intensive dialogues with the family. Despite great workload stress enough time for such conversation should be taken, because the physician will generously be repaid by the way he sees his medical activity. The maintenance of a culture of empathy within the intensive care team is a major task for the leader. In this manner, the ICU will become and remain a place for living humanity.

\section{Keywords}

Medical futility · Decision making · Empathy . Counseling $\cdot$ Patient care team mungsrecht des Patienten ist heute oberstes Gebot; das hat der Gesetzgeber neuerdings nochmals bestärkt.

Allerdings bleiben diese Grundsätze verständlicherweise auf das Generelle begrenzt. In der Intensivmedizin wünschen wir uns konkretere Strategien und Handlungsanweisungen. Deshalb hat die Sektion „Ethik“ der Deutschen Interdisziplinären Vereinigung für Intensiv- und Notfallmedizin (DIVI) ein Positions- papier entwickelt, das die besonderen Belange der Intensivmedizin berücksichtigt [7].

\section{Therapiezielfindung}

Wie finden wir uns aber nun in der schwierigen Frage zurecht, ob wir noch heilen können, ob wir noch heilen müssen? Oder ob wir letztlich unser Therapieziel in Richtung Palliation ändern müssen, 


\section{Infobox 1 Regeln bei Unsicher- heit der Therapieentscheidung zum Lebensende. (Nach Lynn et al. [9])}

- Die sog. infauste Prognose (Aussichtslosigkeit der Therapie) ist meist nicht zweifelsfrei zu bestimmen.

- Es gibt selbst wenige Tage vor Eintritt keine sicheren Zeichen für den bevorstehenden Tod.

- Die Feststellungen „infauste Prognose“ oder "im Sterben" sind daher notwendigerweise subjektiv.

- Daher muss ein Sterben in Würde bereits geplant werden, wenn noch Überlebenschancen bestehen.

- In der klinischen Praxis ist daher ein kombiniertes Vorgehen nötig:

- Behandeln und Kämpfen für das Leben,

- Akzeptanz für das Sterben.

also nur noch Leiden lindern, ohne heilen zu können.

Eines ist klar und eindeutig: Eine Maßnahme ist medizinisch nicht indiziert, wenn im konkreten Fall das angestrebte Therapieziel nicht (oder aber nur mit geringster Wahrscheinlichkeit) erreicht werden kann.

\section{》) Bei Nichterreichbarkeit des Therapieziels ist eine Maßnahme nicht indiziert}

Glauben wir jedoch, dass wir mit unserer Therapie das Ziel einer Heilung erreichen können, dann muss ein solcher Therapieversuch auch dem erklärten Willen des Patienten entsprechen. Es ist aber gerade dieser Patientenwille, der selbst heute noch nicht jederzeit gebührend beachtet wird.

Aufschlussreich dazu ist eine Studie von Sommer et al. [16]: In einer Querschnittuntersuchung in 11 Senioreneinrichtungen stellten sie fest, dass nur $11 \%$ der Insassen eine Patientenverfügung und nur 1,4\% eine Vertreterverfügung hatten. Von119 analysierten Verfügungen erlaubten $52 \%$ keinen Rückschluss auf die Einwilligungsfähigkeit zum Verfügungszeitpunkt, bei nur 3\% war eine ärztliche Beratung dokumentiert. Bei der Prüfung anhand von 5 Behandlungsszenarien gab es meist keine Aussage für den Fall einer akut auftretenden Nichteinwilligungsfähigkeit. In 23 Verfügungen wurde ein Reanimationsversuch bei Herzstillstand zwar ausgeschlossen, jedoch gab es dazu bei 14 Fällen keine entsprechende Absprache mit den Pflegenden.

Die Schlussfolgerung der Autoren war, dass Vorausverfügungen in Senioreneinrichtungen bislang eher selten sind. Sofern sie vorliegen, sind sie wenig aussagefähig und selten valide. Und selbst wenn sie vorliegen, bleiben sie häufig vom Pflegepersonal unbeachtet.

\section{Futility}

Die „futility“, die Erfolglosigkeit, die Sinnlosigkeit unserer ärztlichen Handlungen ist sehr schwer zu beurteilen [3, 14]. Gerade die Erfahrensten von uns kennen Fälle, die wir eigentlich schon aufgegeben haben, die am Ende aber die Station lebend verließen, - während andere, von denen wir sicher waren, sie heilen zu können, es nicht geschafft haben.

Das bedeutet zweierlei:

- Wir müssen immer wieder erneut die aktuelle Situation überprüfen und unsere Entscheidungen hinterfragen.

- Wir müssen uns stets bewusst sein, dass wir uns irren können.

(> Mit einem möglichen Irrtum in der Prognose müssen wir leben.

Unter diesen Bedingungen müssen wir dann diese schwere Entscheidung tragen und ausführen. Wie können wir damit umgehen? Einige wichtige Regeln haben Lynn et al. [9] entwickelt (• Infobox. 1).

Doch die Probleme der Praxis zeigt eine qualitative Interviewstudie aus der Universität Basel [1]: In 52 Interviews mit erfahrenen ärztlichen und pflegerischen Mitarbeitern (17 aus der Intensivmedizin, 18 aus der Langzeitgeriatrie, 17 aus der Akutgeriatrie) wurde die Frage gestellt: „Kennen Sie Situationen, in denen Therapieabbruch bzw. -verzicht sinnvoll gewesen wäre, aber nicht durchgeführt wurde?" Es antworteten 76\% der Ärzte und 86\% der Pflegepersonen mit ja.

Aufschlussreich sind die jeweils genannten Gründe dazu:

- Zeitpunkt verpasst,

- unvollständige Information,
- Dissens zwischen den Ärzten/ hierarchische Entscheidung,

- Dissens zwischen Ärzten und Pflegekräften/unilaterale ärztliche Entscheidung,

- die Ansicht: „In einer Universitätsklinik wird behandelt",

- Defensivmedizin mit Angst vor Unterlassung,

- Ärzte haben Mühe, den Tod zu akzeptieren,

- Verlangen der Angehörigen.

Diese Befunde sind umso bedeutender, als nach einer Studie von Meltzer et al. [10] diese Entscheidungsprobleme eine der Hauptursachen des Burn-out-Syndroms bei Pflegekräften einer Intensivstation sind.

\section{Intensive Zuwendung}

Auf jeden Fall erfordert die letzte Lebensphase auf der Station intensive Zuwendung und wiederholte Gespräche mit den Angehörigen (bzw. den Patienten). Nicht selten gibt es keine Angehörigen, sodass Mitarbeiter im Behandlungsteam sogar die Rolle von „Ersatzangehörigen“ übernehmen müssen [6].

Nun höre ich uns alle sagen:

$\nabla$ In den heutigen Zeiten ist diese Zuwendung so schwierig zu verwirklichen.

Überall werden wir jetzt mit Leistungsdruck konfrontiert. Wir fühlen uns unter Zeitdruck und im ständigen Stress. Wie sollen wir da noch Zeit für lange Gespräche finden? Grom et al. sprechen von einer sog. Industrialisierungskultur, die die Intensivmedizin prägt [6].

Unabhängig davon ahne ich aber, dass dieses vielleicht nicht nur ein Problem von uns Ärzten ist, sondern ein Problem der Gesellschaft insgesamt, ... und wir sind eben Teil dieser Gesellschaft.

\section{Mangel an Zuwendung}

Heute leben wir in einer ichbezogenen Gesellschaft, die die Individualisierung und die grenzenlose Freiheit des Einzelnen, die „Selbstverwirklichung", zum wichtigsten Ziel erklärt hat. In einer sol- 


\section{Infobox 2 Regeln für Angehörigengespräche}

- sich Zeit nehmen: keine Ungeduld (auch nicht nonverbal)

- Aufmerksamkeit: keine Ablenkung, keine abweisende Haltung

- Zuhören - manchmal kann man auch schweigen.

- Verständlich sprechen:

- kein "Medizinerdeutsch"

- keine "großen Sprüche"

- keine Lügen ... aber Tatbestände mit Behutsamkeit darlegen

- Toleranz: Patienten/Angehörige können (dürfen) "nerven"

- Vorwürfe annehmen/Zweitmeinung zulassen

- Ziele setzen: etwa „... wenn nicht bis Donnerstag ..., dann müssen wir ..."

- Nichtwissen zugeben: stärkt erstaunlicherweise das Vertrauen

- Empathie spüren lassen: Zuwendung im wörtlichen Sinne/keine akademische Distanz

chen Gesellschaft bleiben aber oft die sozialen Bindungen auf der Strecke, das Eingebundensein und die Verpflichtung zur Gemeinschaft.

Ein Merkmal dieser ichorientierten Gesellschaft ist der Mangel an Zuwendung, der Mangel an sozialem Mitgefühl, den wir ja überall spüren, - nicht nur in der Medizin.

Diese Erkenntnis wird auch dadurch nicht aufgehoben, dass wir oft eine bemerkenswerte Spendenbereitschaft wahrnehmen (wie jüngsthin bei der Flutkatastrophe); aber vielleicht könnte dieses ja ein Zeichen unseres schlechten Gewissens sein.

Ist unser Mangel an Zuwendung ein gesellschaftstypisches Versagen, ... oder ist es auch Furcht oder Flucht?

Es kann natürlich sein, dass gerade wir Akademiker vor den Emotionen und der Empathie in die Sachlichkeit der Intensivmedizin, der Apparatemedizin, des täglichen Geschehens flüchten. Denn Zuwendung macht schutzlos: Wenn wir mit den Angehörigen über die letzten Dinge sprechen müssen, wenn wir uns wirklich ihnen zuwenden, dann geben wir unser akademisches Schutzschild auf, hinten dem wir uns (als „Halbgott in Weiß") sicher verbergen können.

\section{Die Medizin muss wieder sprechen lernen}

Ich glaube, wir Intensivmediziner müssen auch wieder zu unserer karitativen Grundlage der Medizin zurückkehren. Das sind wir unserem Beruf schuldig; deswegen sind wir doch Ärzte geworden. Sprechen lernen beginnt mit dem Zuhören. Wir Intensivmediziner müssen zunächst lernen, unseren Patienten und ihren Angehörigen wieder zuzuhören und uns auf sie zu konzentrieren.

Wir kennen das doch alle: Wir beherrschen die nonverbale Ausstrahlung perfekt, die da sagt: „Ich habe jetzt keine Zeit“. Wir hören zwar zu, aber gleichzeitig schauen wir auf den Computerbildschirm, weil ja die Befunde so wichtig sind. Wir lassen uns vom Telefon stören, wir schauen ständig nach unserem Smartphone, - denn alles andere ist ja wichtiger als die Kommunikation mit dem Patienten. Aber:

\section{\ Unsere Zuwendung beginnt mit dem Zuhören.}

Wir wissen doch eigentlich alle nur zu gut, wie sehr die Patienten es wertschätzen, wenn der Arzt ihnen Aufmerksamkeit schenkt, ... und doch verstoßen wir täglich gegen diese Regel. Warum wohl ist die alternative Medizin so beliebt (unverdientermaßen, wie ich meine)? Weil sie zuhören kann, weil sie kommunizieren kann. Das sollten wir doch auch können.

\section{Gespräch}

In der Intensivmedizin ist das Patientengespräch eher die Ausnahme; Angehörigengespräche sind dagegen die Regel. Glücklicherweise haben wir meist eine offene Intensivstation, bei der Angehörige frei ihre Patienten besuchen können. Verständlicherweise ist dann der Bedarf groß, mit einem Arzt oder einer Pflegekraft zu sprechen.

So schwierig dieses oft mit dem Ablauf des Routinegeschehens auf der Station zu vereinbaren ist, so sollten wir doch darüber dankbar sein. Diese Gespräche bieten uns die Chance, mit den Angehörigen ein Vertrauensverhältnis aufzubauen, das bei schwierigen Entscheidungen oft hel- fen kann. Außerdem haben wir die Möglichkeit zu zeigen, dass Intensivmedizin eben nicht Apparatemedizin ist, sondern in hohem Maße heilendes Bemühen und Fürsorge bedeutet. Wir sollten also diese Möglichkeiten ausgiebig wahrnehmen.

Für das Angehörigengespräch sollten wir allerdings deutlich mehr Aufmerksamkeit aufbringen, als das oft geschieht. Es sollte nicht beiläufig, zwischen Tür und Angel stattfinden. Bei allem Verständnis für unseren täglichen Stress sollten wir uns als Ärzte klar machen, welche Bedeutung für die Angehörigen ein auch noch so kurzes Gespräch mit dem behandelnden Arzt hat. Einige Regeln bietet $\bullet$ Infobox. 2 .

Tatsächlich ist ein gutes Angehörigengespräch Zuwendung im besten Sinne, insbesondere dann, wenn der Arzt seine akademische Distanz überwinden kann. Außerdem gibt ein gutes Angehörigengespräch dem Arzt selbst eine persönliche Befriedigung, eine berufliche Genugtuung. Ich habe aus persönlichem Erfahren oft wahrnehmen können, dass selbst die schwierigsten und belastendsten Angehörigengespräche mich letztendlich beglückt haben. Die Burn-out-Forscher nennen das positive Resonanz. Vielleicht ist $\mathrm{Zu}$ wendung tatsächlich sogar ein wirksames Prinzip gegen das eigene „Burn-out“: Es stärkt das Selbstwertgefühl.

Wir können und müssen also schon aus unserem eigenen Selbstwertgefühl heraus (also sozusagen aus Egoismus) die mitmenschliche Zuwendung wieder pflegen - gerade in Zeiten der fortschreitenden Ökonomisierung der Medizin.

I) Zuwendung muss gerade in Zeiten der fortschreitenden Ökonomisierung der Medizin gepflegt werden

Jüngsthin hat beim 116. Deutschen Ärztetag in Hannover der Freiburger Medizinethiker Prof. Dr. Giovanni Maio die Ärzte davor gewarnt, dass sie sich umprogrammieren lassen: Er sieht die Gefahr, dass die Ärzte „... Zug um Zug die eigentlich fremde Logik der Ökonomie zu ihrer eigenen Logik machen". In dieser Logik aber ist Zuwendung nicht vorgesehen. 
Um unserer eigenen Ideale willen sollten wir also die Zuwendung hochhalten.

Und in der Sicht von außen, aus der Sicht der Gesellschaft stehen wir doch ohnehin schon im Verdacht: Gerade in der kostenträchtigen Intensivmedizin kann der Verdacht rasch aufkommen, dass beim Beenden einer Behandlung nur Kosten gespart werden sollen. Also müssen wir unsere Entscheidungen sorgfältig erklären; so erklären, dass der Angehörige und Laie es wirklich versteht. Sie müssen voll auf unserer Seite stehen. Das gilt v. a. für den Therapiezielwechsel zur Palliativmedizin.

\section{》) Angehörige brauchen unsere Hilfe}

Eines ist auf jeden Fall gewiss: Wir Ärzte dürfen die Angehörigen in ihrer schweren Entscheidung nicht alleine lassen. Angehörige brauchen unsere Hilfe.

\section{Kultur der Zuwendung}

Ich meine, es gibt eine Kultur der Zuwendung (so wie es leider auch auf diesem Gebiet oft die Kulturlosigkeit gibt). Und es ist die Aufgabe des Leiters, diese Kultur der Zuwendung aufzubauen: Er setzt die Zeichen. Er bestimmt die Richtung. Er ist das Vorbild. Von ihm muss die Aufgeschlossenheit zur Zuwendung ausgehen, er muss sie unterstützen, möglichst sogar vorleben.

Die Basis für eine Kultur der Zuwendung ist aber das Team: Ein gutes Intensivteam ist auf Vertrauen und gegenseitigem Respekt zwischen den verschiedenen Partnern des Pflegeteams und den ärztlichen Mitarbeitern aufgebaut. Jeder bringt seine unbedingte Motivation und seine Expertise ein und verdient deswegen von den Partnern Anerkennung. Schließlich hat die Pflege einen wesentlichen, wenn nicht sogar überwiegenden Anteil an dem Erfolg unserer Intensivbehandlung.

\section{Teamkultur}

$\mathrm{Zu}$ der Kultur der Zuwendung gehört auch die Teamkultur. Wir kennen alle die unterschiedliche Wahrnehmung zwi- schen Ärzten und Pflegekräften in diesen letzten Fragen.

Der Arzt fühlt sich als Wissenschaftler; er steht für Heilkunst, Wissen, evidenzbasierte Medizin. Die Pflegenden aber fühlen sich nahe am Menschen; sie stehen für Fürsorge und Empathie. Sie haben den besseren Blick für die Bedürfnisse und Probleme ihrer Patienten.

Wir Ärzte täten also gut daran, uns bei der Zuwendung zum Patienten (und zu den Angehörigen) von erfahrenen Pflegekräften an die Hand nehmen zu lassen. Gerade bei den Besprechungen über die letzten Dinge, bei den Entscheidungen zur Therapiebegrenzung und zur Therapiezieländerung, müssen die Pflegekräfte ihre Ansichten und Überzeugungen offen darlegen dürfen; sie müssen bei uns Ärzten Gehör finden und respektiert werden.

Oft aber fühlen sie sich in ihrer Einschätzung von den Ärzten nicht beachtet. Das führt zu Frustrationen im Team [2]. Hier ist Teamkultur gefragt, die v. a. der Leiter anregen muss. Erst wenn Offenheit und Ehrlichkeit im Team gelebt werden, erst dann ist es wirklich ein Team.

Dieser Teamgedanke muss bewusst trainiert werden. Das benötigt Zeit und ständige regelmäßige Gespräche. Das bedeutet aber auch, dass das Team über längere Dauer personell stabil bleiben muss. Bei einem ständigen Personalwechsel ist kein Team aufzubauen.

Der Aufbau eines mündigen Teams bedeutet insbesondere auch, die eigene Meinung einzelner Mitglieder zu respektieren, - auch wenn sie kontrovers ist. Das setzt voraus, dass sie ermutigt werden, ihre eigene Meinung auszudrücken. Auch hier muss wieder der Leiter mit konsequentem Vorbild vorangehen. Nicht selten geht die Unterdrückung kontroverser Meinungen ja von ihm aus (das übliche „Chefgebahren“); dabei sollte er auf ein Team stolz sein, das eigene konstruktive Vorschläge hervorbringt. Es sind gerade die Kontroversen, die ein Team weiterbringen. Sie müssen allerdings ausdiskutiert werden. Insbesondere bei Minderheitsansichten aus der Pflege müssen wir Ärzte sehr sensibel reagieren. Wie leicht neigen wir Akademiker dazu, überheblich zu wirken, selbst wenn wir es vielleicht gar nicht so meinen. Nichts stärkt die Effektivität eines Teams so, wie das
Bewusstsein der eigenen Qualität, wie ein berechtigtes Selbstbewusstsein im Team. Dabei muss der Leiter allerdings auch die Gefahren eines überzogenen Gruppendenkens kennen, das sehr rasch Andersdenkende mundtot macht (s. [8]).

Es ist ein langer Prozess zum gegenseitigen Verstehen, zum gegenseitigen Respekt. Aber es lohnt sich. Wenn diese Grundsätze der Menschlichkeit in dem Intensivteam gelebt werden, dann dürfen wir auch zuversichtlich hoffen, dass die jüngere Generation sich wieder für die Intensivmedizin begeistern lässt und sich in Zukunft - trotz der hohen Belastung voll für dieses faszinierende Fach einsetzt.

\section{Schlussbemerkung}

Die Grundlagen zu meinen Überlegungen basieren natürlich auf meinen eigenen beruflichen Erfahrungen. Die liegen jetzt gut 10 Jahre zurück. Vielleicht hat sich ja schon in der jüngst vergangenen Zeit einiges getan. Ich bekomme diesen Eindruck, wenn ich heute mit Intensivmedizinern spreche: Wir sind reifer geworden, akzeptieren unsere Ohnmacht. Wir sind vielleicht ein wenig demütiger geworden und haben erkannt, dass wir auch lernen müssen, loszulassen. Und hier können Intensiv- und Palliativmedizin Hand in Hand gehen.

Die Palliativmedizin entlastet uns Intensivmediziner von dem gefühlten Zwang, immer erfolgreich sein zu müssen. Die Palliativmedizin könnte das gute Gewissen der Intensivmedizin werden. Denn: Die Intensivstation muss ein Ort der Menschlichkeit bleiben.

\section{Fazit für die Praxis}

- Sachlichkeit und Effizienz prägen die moderne Intensivmedizin. Sie haben zu zählbaren Erfolgen geführt. Unter dem enormen Leistungsdruck kommen allerdings Zuwendung und Empathie zu kurz.

- Die Behandelnden der Intensivstation sollten ihren Patienten mehr emotionale Zuwendung bieten und den Angehörigen mit verständnisvollen Gesprächen helfen, die belastende Leidenssituation zu meistern. Dabei ist auch empathisches Zuhören erfor- 
derlich, nicht nur die Vermittlung von informativen Tatbeständen.

- Diese Kultur der Zuwendung muss vom Leiter getragen und vorgelebt werden.

- Bei Erfolglosigkeit der intensivmedizinischen Behandlung muss das Therapieziel hin zur palliativen Leidenslinderung geändert werden. Das ist die konsequente Fortsetzung der intensivmedizinischen Bemühungen.

\section{Korrespondenzadresse}

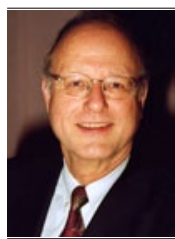

Prof. Dr. H. Burchardi, FRCA

Kiefernweg 2,

37120 Bovenden

hburcha@gwdg.de
10. Meltzer LS, Huckabay LM (2004) Critical care nurses' perceptions of futile care and its effect on burnout. Am J Crit Care 13:202-208

11. Müller-Busch HC (2013) Palliativmedizin und Sterben auf der Intensivstation - kein Widerspruch. DIVI 4:22-27

12. Nauck F, Dlubis-Mertens K (2011) Germany has adopted a charter for the care of the critically ill and the dying. Eur J Palliat Care 18:176-178

13. Nauck F, Jaspers B (2012) Vom intensivmedizinischen zum palliativmedizinischen Behandlungsziel. In: Putensen C, Quintel M, Sybrecht GW (Hrsg) DIVI Jahrbuch 2011/2012. MWV, Berlin, S 369-375

14. Schneidermann $L$, Jecker N, Jonsen A (1990) Medical futility: its meaning and ethical implications. Ann Intern Med 112:949-954

15. Schulze MC (2013) Wider den Absicherungswahn. Multimorbide Patienten. Dtsch Ärztebl 110:A124A125

16. Sommer S, Marckmann G, Pentzek M et al (2012) Patientenverfügungen in stationären Einrichtungen der Seniorenpflege. Dtsch Ärztebl 109:577583

\section{Einhaltung ethischer Richtlinien}

Interessenkonflikt. H. Burchardi erklärt, dass kein Interessenskonflikt besteht.

Dieser Beitrag beinhaltet keine Studien an Menschen oder Tieren.

\section{Literatur}

1. Albisser Schleger $H$, Pargger $H$, Reiter-Theil $S$ (2008) „Futility" - Übertherapie am Lebensende? Gründe für ausbleibende Therapiebegrenzung in Geriatrie und Intensivmedizin. Z Palliativmed 9:67-75

2. Azoulay E, Timsit JF, Sprung CL et al (2009) Prevalence and factors of intensive care unit conflicts. The conflicus study. Am J Respir Crit Care Med 180:853-860

3. Becker G, Blum HE (2004) „Medical Futility“: Der Arzt im Spannungsfeld von Behandlungsauftrag und Behandlungsbegrenzung. Dtsch Med Wochenschr 129:1694-1697

4. Bundesärztekammer (2004) Grundsätze der Bundesärztekammer zur ärztlichen Sterbebegleitung. Dtsch Ärztebl 101:A1298-A1299

5. Bundesärztekammer (2011) Grundsätze der Bundesärztekammer zur ärztlichen Sterbebegleitung. Dtsch Ärztebl 108:A346-A348

6. Grom IU, Vagts DA, Kampa U et al (2013) Patienten am Lebensende auf der Intensivstation. Anaesthesist 62:473-482

7. Janssens U, Burchardi H, Duttge G et al (2012) Therapiezieländerung und Therapiebegrenzung in der Intensivmedizin. DIVI 3:103-107

8. Janis IL, Mann L (1977) Decision making. A psychological analysis of conflict, choice and commitment. Free Press, New York

9. Lynn J, Harrell F Jr, Cohn F et al (1997) Prognoses of seriously ill hospitalized patients on the days before death: implications for patient care and public policy. New Horiz 5:56-61

\section{Arteriosklerose-Artikel online frei zugänglich}

Hierzulande ist Arteriosklerose eine der häufigsten Gefäßerkrankungen; 18\% der Frauen und $28 \%$ der Männer in der Altersgruppe über 65 Jahre sind betroffen. Im Zuge der ACTION (Arteriosklerose -

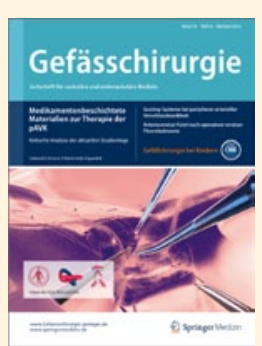
Circulation und Training InformatiOns Netzwerk)Kampagne der Deutschen Gesellschaft für Gefäßchirurgie und Gefäßmedizin (DGG) stehen zwei

Übersichtsarbeiten zu Arteriosklerose für einen breiten Leserkreis online frei zugänglich bereit.

Im ersten Beitrag in Ausgabe 6/13 von Gefässchirurgie beschreiben die Autoren Risikofaktoren, Epidemiologie und die Ursachen von Arteriosklerose, im zweiten Beitrag in Ausgabe 7/13 von Gefässchirurgie erläutern sie die Manifestation der Arteriosklerose und die Bedeutung von Präventionsmaßnahmen.

\section{Ursachen und Risikofaktoren der} Arteriosklerose

dx.doi.org/10.1007/s00772-013-1233-6

\section{Manifestation und Prävention der} Arteriosklerose dx.doi.org/10.1007/s00772-013-1235-4

Mit der ACTION -Kampagne trägt die Deutsche Gesellschaft für Gefäßchirurgie und Gefäßmedizin (DGG) zur Aufklärung, Information und Prävention über Arteriosklerose bei.

Suchen Sie noch mehr zum Thema? Mit e.Med, dem Online-Paket von Springer Medizin, können Sie schnell und komfortabel in über 500 medizinischen Fachzeitschriften recherchieren.

Weitere Infos unter: springermedizin.de/eMed. 